\title{
Electrochemical Deposition of ZnO Nanorod Arrays onto a ZnO Seed Layer: Nucleation and Growth Mechanism
}

\author{
M. Romero ${ }^{1, *}$, R. Henríquez ${ }^{1}$, E. A. Dalchiele ${ }^{2}$ \\ ${ }^{1}$ Instituto de Química, Facultad de Ciencias, Pontificia Universidad Católica de Valparaíso, Av. \\ Universidad 330, Curauma, Placilla, Valparaíso, Chile. \\ ${ }^{2}$ Instituto de Física\& CINQUIFIMA, Facultad de Ingeniería, Herrera y Reissig 565, C.C. 30, 1100 \\ Montevideo, Uruguay. \\ *E-mail: marioromero290@gmail.com
}

doi: $10.20964 / 2016.10 .61$

Received: 11 July 2016 / Accepted: 24 August 2016 / Published: 6 September 2016

The nucleation and growth mechanisms for the electrochemical synthesis of $\mathrm{ZnO}$ nanorod arrays on two different substrates (i.e., a naked FTO/glass substrate and a ZnO thin film seed layer/FTO/glass substrate) were investigated. This study was based on the analysis and deconvolution of the chronoamperometric transient curves that were obtained during the potentiostatic electrosynthesis of $\mathrm{ZnO}$ nanostructures. Using this potentiostatic method and existing theoretical formalism, the nucleation kinetic parameters were determined. Analysis of these results indicated that the presence of a seed layer did not change the nucleation or growth mechanisms and only affected the time constant of each contribution (i.e., 3D instantaneous nucleation with diffusion controlled growth and 3D instantaneous nucleation under charge-transfer control) as well as the associated Faradic charge.

Keywords: Nanorod, electrodeposition, NGM, ZnO.

\section{FULL TEXT}

(C) 2016 The Authors. Published by ESG (www.electrochemsci.org). This article is an open access article distributed under the terms and conditions of the Creative Commons Attribution license (http://creativecommons.org/licenses/by/4.0/). 